\title{
A High Resolution Survey of OH Masers in the Southern Hemisphere with a Parasitic SETI Program
}

\author{
Guillermo A. Lemarchand \\ Instituto Argentino de Radioastronomía (CONICET) and Centro de \\ Estudios Avanzados(UBA).E-mail:lemar@cea.uba.ar
}

\begin{abstract}
We describe the ongoing high and ultrahigh-resolution survey of $\mathrm{OH}$ masers in the southern hemisphere. The study is made using one of the 30-m antennas of the Argentine Institute for Radioastronomy (IAR) connected simultaneously to two different spectrometers: (a) one 1008 channel correlator with a spectral resolution of 33 to $312 \mathrm{~Hz}$, and (b) an eight million channel analyzer with a spectral resolution of $0.05 \mathrm{~Hz}$ (META II). The observations are performed with a high-gain feed with $35 \mathrm{~K}$ system temperature. The preliminary survey is concentrated within a list of 24 strong $\mathrm{OH}$ masers at 1665 and $1667 \mathrm{MHz}$. The purpose of our research is to test theoretical considerations about the mechanisms of temporal variation in $\mathrm{OH}$ masers as well as the possible use of $\mathrm{OH}$ masers as natural amplifiers of extraterrestrial artificial signals.
\end{abstract}

\section{Introduction}

Theoretical work (Scappaticci \& Watson 1992a,b) predicts the variability of OH masers with times of $1000 \mathrm{sec}$. Our search is trying to find this variability as well as to determine the spectral fine structure of some of these masers. These variations may occur when (1) the maser is partly but not heavily saturated, (2) when the decay rate for the molecular states is near $c / L$ (where $c$ is the speed of light and $L$ is the length of the maser) and (3) when the product of the brightness temperature $T_{o}$ of the incident radiation and the angle for the beaming is less than a critical value that depends upon the particular masing radiation. A fourth parameter -the fractional inversion in the pumping multiplied by $\left(T_{o} /\right.$ frequency)- determines the importance of spontaneous emission which can eliminate the instabilities. These instabilities are a likely cause for the fluctuations in the radiation from the $18 \mathrm{~cm} \mathrm{OH}$ masers that are estimated to occur on time scales as short as 1000 sec. One purpose of this work is to try to detect this theoretical behavior within our $\mathrm{OH}$ preliminary list.

At the same time, the META II system (Lemarchand et al. 1996) is analyzing the same $\mathrm{OH}$ maser signals, searching for artificial extraterrestrial transmissions that may be beamed through the cosmic maser regions. Cordes (1993) and Lemarchand (2000) have shown that this may be an extraordinary advantage for interstellar communications strategies, because we may combine several unknowns of the SETI cosmic haystack within the same search. For example, this may be a criterion to select a possible magic frequency $(1665$ and $1667 \mathrm{MHz})$ as 


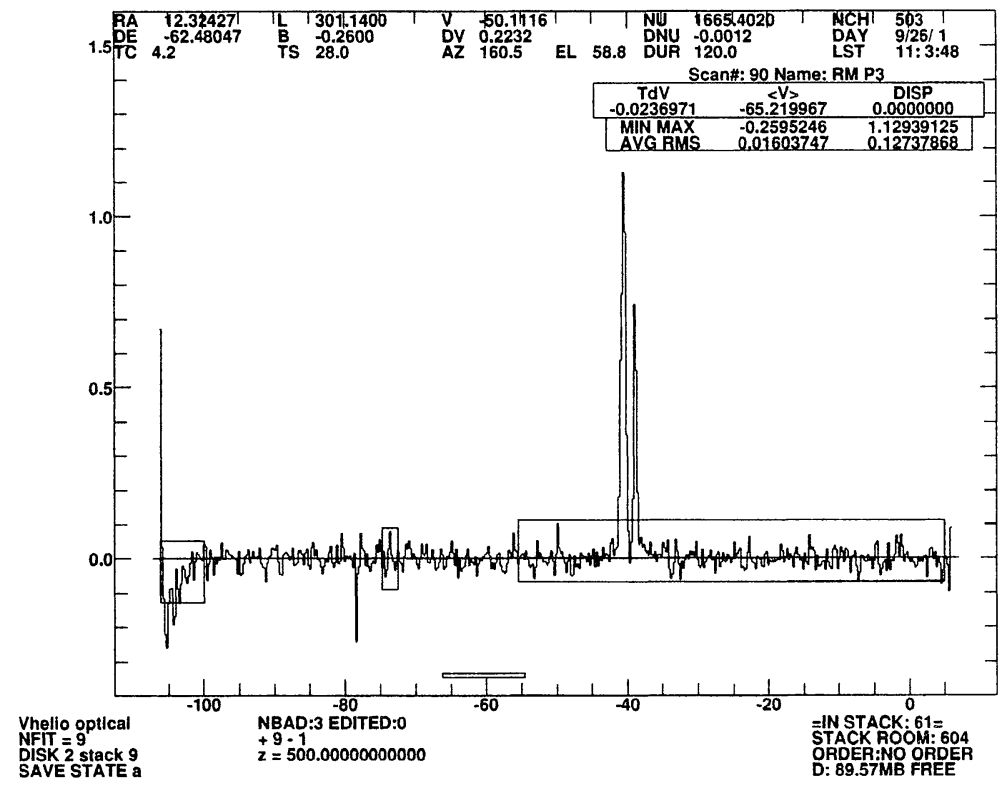

Figure 1. Typical high resolution profile of an $\mathrm{OH}$ maser. In this case the spectral resolution is $312 \mathrm{~Hz}$.

the resonant frequencies of masers, combined with the directions in the sky (location of cosmic masers), combined with higher gains due to the natural maser amplification effect $\left(10^{7}\right.$ to $\left.10^{11}\right)$. To test all these hypotheses we use an ultra high resolution $(0.05 \mathrm{~Hz})$ spectrum analyzer.

\section{Observations}

The observations started April 2002. We have selected 24 different $\mathrm{OH}$ masers at 1665 and $1667 \mathrm{MHz}$, some of them with several maser components. These masers were originally discovered in surveys made by Turner (1979) and Caswell \& Haynes $(1980,1983)$. All these objects are located in the southern hemisphere (declinations between -10 and -90 degrees).

Figure 1 shows a typical profile after $10 \mathrm{~min}$. of integration time. Each maser is observed for four hours, making partial integrations each minute. We use a 1008 channel correlator with a spectral resolution ranging from 33 to $312 \mathrm{~Hz}$. These resolutions are below the limit of $550 \mathrm{~Hz}$ that corresponds to the most narrow maser reported in the literature (Cohen et al. 1987). The observations are repeated each 6 months in order to detect possible variations in their intensity and changes in the fine structure of their spectrum. As yet, we 
have been unable to find any significant variation in the intensity as predicted by theoretical models.

\section{The Use of Natural Masers Amplifying Capabilities as a SETI Strategy}

Cordes (1993) made a careful study of the possible use of masers for interstellar communication. He concluded that the most reasonable strategy is to perform an intensive survey of the galactic plane to sample many maser sites. This strategy is compatible with traditional astronomical studies of the galactic plane (Turner 1979; Caswell \& Haynes 1980, 1983). If our preliminary OH observations obtains reasonable results for maser timing variations, these results will justify the startup of a very long term galactic plane survey to be done at IAR. An approximation of a full plane survey would be to choose directions where star formation or the density of late-type stars is most prevalent, ie. the inner Galaxy.

The previous high resolution study of $\mathrm{OH}$ masers was done by Cohen et al.(1987). Observing OH/IR stars they have found natural masers features with widths $>500 \mathrm{~Hz}$, consistent with theoretical considerations about masing mechanisms. The artificial extraterrestrial signals are hypothesized to be much narrower (several $\mathrm{Hz}$ ). For this reason we are observing simultaneously with two different spectrometers: (a) a 1008 channel correlator with 33 to 312 $\mathrm{Hz}$ of spectral resolution and (b) an 8.4 million channel with $0.05 \mathrm{~Hz}$ spectral resolution.

Interstellar scattering will cause the input signal to the maser, as well as the output to be modulated by $100 \%$. Scintillations may be countered effectively by making several separate observations of the maser regions in order that one of them occurs when scintillations modulate the source to stronger than average strength (Cordes \& Lazio 1991). The hypothetical transmitting civilization would combat scintillation by beaming towards a given maser for a time greater than many characteristic scintillation time or for a sequence of short times separated by more than one characteristic scintillation time (hours). These boundary conditions are satisfied by our observation scheme: (i) taking high resolution integration times each $20 \mathrm{sec}$ and (ii) observing each source for at least four hours in each run.

Acknowledgments. The SETI project in Argentina, of which this research is part, is supported by The Planetary Society. My participation in this meeting was possible thanks to the generosity of Allen Tough and an IAU travel grant.

\section{References}

Caswell, J. L., \& Haynes, R. F. 1980. Aust. J. Phys., 33, 639

Caswell, J. L., \& Haynes, R. F. 1983, Aust. J. Phys., 36, 361

Cohen, R. J., et. al. 1987, MNRAS, 225, 491

Cordes, J. M. 1993, ASP Conf. Ser. 47, 257

Cordes, J. M., \& Lazio, J. 1991, ApJ, 376, 123

Lemarchand, G. A. 2000, ASP Conf. Ser., 213, 467 
Lemarchand, G. A., Colomb, F. R., Hurrell E. E., \& Olalde, J. C. 1996, in Proc. of IAU Colloquium 161, ed. C. Cosmovici, S. Bowyer \& D. Werthimer, (Bologna: Editrice Compositori), 611

Scappaticci, G. A., \& Watson, W. D. 1992a, ApJ, 400, 351

Scappaticci, G. A., \& Watson, W. D. 1992b, ApJ, 387, L73

Turner, B. E. 1979, A\&AS37, 1

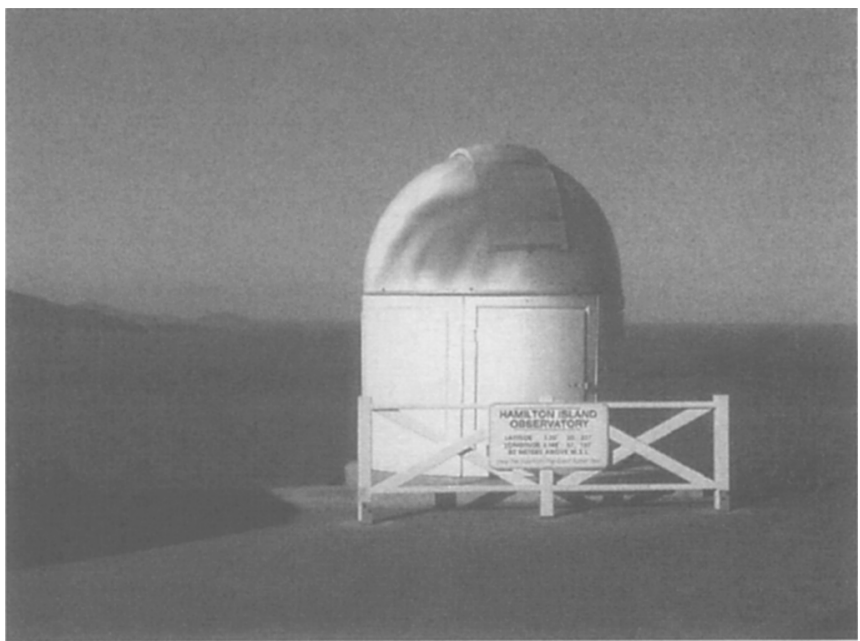

Ray Johnston's observatory on Hamilton Island (photo: Jan Soldan) 